IMSc/95-96/001

\title{
Solar and atmospheric neutrino oscillations with three flavours
}

\author{
Mohan Narayan $^{a}$, M.V.N.Murthy ${ }^{a}$, G. Rajasekaran ${ }^{a}$ and S. Uma Sankar ${ }^{a, b}$ \\ ${ }^{a}$ Institute of Mathematical Sciences, Madras 600 113, India. \\ ${ }^{b}$ Department of Physics, Indian Institute of Technology, Bombay
}

(October 8, 2018)

\begin{abstract}
We analyze the solar and the atmospheric neutrino problems in the context of three flavour neutrino oscillations. We assume a mass hierarchy in the vacuum mass eigenvalues $\mu_{3}^{2} \gg \mu_{2}^{2} \geq \mu_{1}^{2}$, but make no approximation regarding the magnitudes of the mixing angles. We find that there are small but continuous bands in the parameter space where the constraints imposed by the current measurements of ${ }^{71} \mathrm{Ga},{ }^{37} \mathrm{Cl}$ and Kamiokande experiments are satisfied at $1 \sigma$ level. The allowed parameter space increases dramatically if the error bars are enlarged to $1.6 \sigma$. The electron neutrino survival probability has different energy dependence in different regions of the parameter space. Measurement of the recoil electron energy spectrum in detectors that use $\nu-e$ scattering may distinguish between some of the allowed regions of parameter space. Finally we use the results for the parameter space admitted by the solar neutrinos as an input for the atmospheric neutrino problem and show that there exists a substantial region of parameter space in which both problems can be solved.
\end{abstract}

PACS numbers: 14.60.Gh, 96.60.Kx, 95.30.Cq, 96.40.Tv 


\section{INTRODUCTION}

The solar neutrino problem has been an interesting and intriguing phenomenon in neutrino physics for a long time. The different solar neutrino experiments observe differing

fractions of the neutrino flux predicted by the Standard Solar Model (SSM) [舟,2]. The oldest of the solar neutrino experiments is the ${ }^{37} \mathrm{Cl}$ experiment at Homestake. Its energy threshold is $0.814 \mathrm{MeV}$ and it can detect the neutrinos from ${ }^{7} \mathrm{Be}\left(E_{\nu}=0.862 \mathrm{MeV}\right)$ and ${ }^{8} B$ $\left(E_{\nu} \leq 14.02 \mathrm{MeV}\right)$ reactions. In the standard solar model (SSM) of Bahcall-Pinnsonneault [3], the capture rate in the ${ }^{37} \mathrm{Cl}$ experiment is predicted to be $8.0 \pm 1.0 \mathrm{SNU}$. However, the measured rate is only [4]

$$
R_{C l}=2.55 \pm 0.25 \mathrm{SNU}
$$

The water Cerenkov detector at Kamioka, with a threshold of $7.5 \mathrm{MeV}$, can detect only the neutrinos from the upper end of ${ }^{8} B$ spectrum and the Kamioka result [5] is,

$$
y_{\text {Kam }}=\frac{R_{\text {Kam }}}{R_{\text {Kam:SSM }}}=0.51 \pm 0.07
$$

which is the ratio of the observed neutrino flux to that predicted by the SSM. The gallium experiments SAGE and GALLEX, with energy threshold of $0.233 \mathrm{MeV}$ can detect the neutrinos coming from the dominant $p-p$ reaction $\left(E_{\nu} \leq 0.42 \mathrm{MeV}\right)$ as well as the neutrinos from ${ }^{7} B e$ and ${ }^{8} B$ reactions. Their measured rates are [6, 7]

$$
\begin{aligned}
R_{S A G E} & =69 \pm 11 \pm 6 \mathrm{SNU} \\
R_{\text {GALLEX }} & =79 \pm 10 \pm 6 \mathrm{SNU}
\end{aligned}
$$

and the average is

$$
R_{\text {Ga;avg }}=74 \pm 8 \mathrm{SNU}
$$

as opposed to the SSM prediction of 131.5 SNU.

A rough model independent analysis of these results indicates that the low energy neutrinos from the $p-p$ reaction suffer very little suppression whereas the higher energy neutrinos 
are suppressed to a large extent [1,8]. Recently it was pointed out that if neutrinos have no properties beyond those in the standard electro-weak model (i.e. if they are massless), the measurement of Kamiokande, together with that of ${ }^{37} \mathrm{Cl}$ experiment, implies that the ${ }^{7} \mathrm{Be}$ neutrinos must be suppressed by more than $90 \%$ [9,10].

In addition there exists an anomaly in the ratio of observed muon neutrinos to electron neutrinos in the earth's atmosphere. These neutrinos are produced from the decay of $\pi^{ \pm}$ and $K^{ \pm}$which are in turn produced by cosmic rays interacting with the atmosphere. The ratio is roughly two as suggested by the Monte-Carlo calculations whereas both Kamioka 11] and IMB [12] report that the ratio is only about half of that predicted by the MonteCarlo calculations. The results for this ratio are also available from three other groups using the tracking detectors, namely the NUSEX [13], Frejus [14 and SOUDAN-II [15] collaborations. The data from the NUSEX collaboration seems to be in agreement with noanomaly situation. Similar conclusion is obtained from Frejus data if all the contained events are considered. However, if only fully contained results are taken into consideration, there is a suppression. The SOUDAN-II results are consistent with the results obtained with water Cerenkov detectors. It should be noted that the statistics in the tracking experiments is not as high as the water Cerenkov experiments. Evidently any solution of the solar neutrino puzzle must incorporate simultaneously a solution of the atmospheric neutrino problem [16].

A satisfactory solution to the solar neutrino problem should be able to explain not only the total deficit that is observed but the differential suppression observed at low and high energies. Solutions based on astrophysics or nuclear physics ascribe the deficit to smaller solar core temparature or smaller cross sections for the nuclear reactions talking place in the sun. Recent model independent analyses suggest that these solutions cannot describe the results of ${ }^{37} \mathrm{Cl}$ and Kamiokande simultaneously [2,8]. Particle physics based solutions attempt to account for the deficit by assuming that the neutrinos have interactions beyond those of the standard electro-weak model. If the neutrinos possess small mass, an electron neutrino can oscillate into a neutrino of another flavour [17]. The amplitude of oscillation is a function of the mass squared differences, the mixing angles between neutrino flavours 
and the neutrino energy. If one of the mass square differences is of the order of the effective mass squared arising from $\nu_{e}-e$ interaction, the matter effects can enhance the mixing to its maximal value and the amplitude for $\nu_{e}$ oscillating into another flavour will be very large [18]. This is the so called MSW effect.

Matter-enhanced oscillations have been studied thoroughly in the scenario where only two flavours, $\nu_{e}$ and $\nu_{\mu}$, mix with each other [19 21]. The vacuum oscillation here is controlled by the two parameters, the mass square difference $\delta_{21}=m_{2}^{2}-m_{1}^{2}$ and the mixing angle $\omega$. Matter effect is taken into account by adding to the mass squared of $\nu_{e}$, the term

$$
A(r)=\sqrt{2} G_{F} n_{e}(r) \times 2 E,
$$

which is proportional to the electron number density in the Sun $n_{e}(r)$, where $r$ is the radial distance from the centre of the Sun. The maximum value of $A$ occurs at the core and is roughly $10^{-5} E \mathrm{eV}^{2}$, where $E$ is the neutrino energy in $\mathrm{MeV}$. The mixing angle $\omega_{m}$ in the presence of matter is given by,

$$
\cos 2 \omega_{m}=\frac{\delta_{21} \cos 2 \omega-A}{\sqrt{\left(\delta_{21} \cos 2 \omega-A\right)^{2}+\left(\delta_{21} \sin 2 \omega\right)^{2}}} .
$$

The MSW resonance condition is,

$$
A=\delta_{21} \cos 2 \omega
$$

Note that, if the resonance condition is to be satisfied, $A_{\text {core }}>\delta_{21} \cos 2 \omega$, which implies that $\omega_{m}>\pi / 4$ at core. At resonance it becomes $\pi / 4$ and approaches its vacuum value after passing through the resonance.

The probability for an electron neutrino produced in the solar core to be detected as an electron neutrino on earth, averaged over the time of emission and the time of absorption, is given by

$$
\left\langle P_{e e}\right\rangle=\cos ^{2} \omega \cos ^{2} \omega_{m}+\sin ^{2} \omega \sin ^{2} \omega_{m}-x_{12} \cos 2 \omega \cos 2 \omega_{m}
$$

where $\omega_{m}$ is to be evaluated at the point of production and $x_{12}$ is the probability of a nonadiabatic jump between the matter dependent mass eigenstates. If the variation of the solar density in the resonance region is slow enough, the adiabatic condition 


$$
\gamma \equiv \frac{\delta_{21}}{E\left|\frac{1}{A} \frac{d A}{d r}\right|_{r e s}} \frac{\sin ^{2} 2 \omega}{\cos 2 \omega} \gg 1
$$

is satisfied and the matter dependent mass eignestates evolve adiabatically and there are no transitions between them. If (8) is not satisfied, then there will be non-adiabatic transitions between the two matter dependent mass eigenstates in the resonance region and the probability of this jump has the general form $\exp (-C / E)$. The term $C$ has dimensions of energy and is some function of $\delta_{21}, \omega$ and the derivative of the solar density. The expressions for $C$ for various density profiles are tabulated in Ref. [22]. For linear density variation in the resonance region, the jump probability is given by the Landau-Zener formula

$$
x_{12}=\exp \left[-\frac{\pi}{2} \gamma\right]
$$

The predictions for the rates of various experiments are obtained by convoluting the SSM neutrino fluxes with the expression for survival probability in (7). A fit to the data from ${ }^{71} \mathrm{Ga},{ }^{37} \mathrm{Cl}$ and Kamiokande experiments yields solutions in two regions in the $\delta_{21}-\sin ^{2} 2 \omega$ plane, one with small vacuum mixing and one with large vacuum mixing:

$$
\begin{aligned}
& \delta_{21} \simeq 6.1 \times 10^{-6} \mathrm{eV}^{2} \text { and } \sin ^{2} 2 \omega \sim 0.0065 \\
& \delta_{21} \simeq 9.4 \times 10^{-6} \mathrm{eV}^{2} \text { and } \sin ^{2} 2 \omega \sim 0.62
\end{aligned}
$$

In case of the small mixing angle solution, the resonance occurs for neutrinos with energy greater than $0.6 \mathrm{MeV}$. Therefore, the $p-p$ neutrinos (whose maximum energy is $0.42 \mathrm{MeV}$ ) are unaffected whereas the neutrinos with energy greater than $0.6 \mathrm{MeV}$ are almost completely converted into $\nu_{\mu}$. But the measurement of Kamiokande shows that the neutrinos with energy greater than $7.5 \mathrm{MeV}$ are suppressed by only a factor of 0.5 . This can be accommodated through the non-adiabatic jump $x_{12}$ in (7). If $C \simeq 10 \mathrm{MeV}$, or equivalently $\delta_{21} \sin ^{2} 2 \omega \sim 4 \times 10^{-8} \mathrm{eV}^{2}$, then $x_{12}$ is negligible for energies less than $5 \mathrm{MeV}$, but becomes appreciable at higher energies and $\left\langle P_{e e}\right\rangle$ satisfies Kamiokande constraint. The energy dependence of $\left\langle P_{e e}\right\rangle$ in this case is precisely of the form that is required to satisfy the data from the three solar neutrino experiments. In the case of the large angle solution, the non-adiabatic 
effects are totally negligible and the $\left\langle P_{e e}\right\rangle$ is about 0.55 below $0.5 \mathrm{MeV}$ and slowly falls to about 0.35 around $5 \mathrm{MeV}$ after which it it remains almost independent of the neutrino energy.

In the case of two flavour oscillations, the area of the parameter space, which can satisfy all the three constraints at $1 \sigma$ level, is very small. Especially, in the case of the small angle solution, the requirement that the resonance should occur around $0.6 \mathrm{MeV}$ uniquely fixes the value of $\delta_{21}$. The requirement that the ${ }^{7} \mathrm{Be}$ neutrinos should be completely suppressed and that the high energy ${ }^{8} B$ neutrinos should have a suppression of about 0.5 determines the product $\delta_{21} \sin ^{2} 2 \omega$ almost exactly. Therefore, there is very little leeway in the allowed values of $\delta_{21}$ and $\sin ^{2} 2 \omega$. An appreciable region of parameter space is allowed only at 95\% C.L. ( or $2.4 \sigma$ level). In addition, this simple picture is inadequate to simultaneously explain the solar and atmospheric neutrino deficits since the mass squared differences required are in vastly different regimes. To explain the atmospheric neutrino anomaly on the basis of two-flavour vacuum oscillations, one requires a mass squared difference of the order of $10^{-1}-10^{-3} \mathrm{eV}^{2}$, with a large mixing angle. This must be compared with the best fit to the data in the case of solar neutrino problem given in eq.(10). Therefore one has to necessarily consider the scenerio in which all the three neutrinos participate. This of course is also a more realistic situation since the LEP experiments have already pinned down the number of light neutrino generations to be three.

Three flavour oscillations were considered previously 23 25]. However, the uncertainties in the Gallium experiments have come down significantly in recent times and the parameter region allowed by the current data will be much smaller. Recently Joshipura and Krastev [26] have attempted a complete solution of the solar and atmospheric neutrino problems in the three generation frame work. They present a combined analysis of these two problems in the framework of the MSW effect and indeed show that there exists a parameter space in which both sets of data can be reconciled. Kim and Lee [27] analyse these two problems and present a solution based on maximally mixed( in vacuum) three generations of neutrinos. This later analysis is however a rather fine tuned solution since the parameter space allowed 
is rather tiny.

In this paper, we analyze the solar neutrino problem by considering the oscillations between the three neutrino flavours. The analysis is done with no particular model of neutrino masses and mixings assumed. The analysis is similar in spirit to that of Joshipura and Krastev [26]. We carry their analysis further and not only map out the full parameter space, but also discuss the average survival probability and recoil electron spectrum. In addition we also discuss a non-standard solution where no resonance occurs but nevertheless there is a parameter space in which all the three experiments discussed earlier can be reconciled. We also do not make any assumption about the evolution being adiabatic and take into account non-adiabatic effects. These effects may be ignored, however, in parts of allowed parameter space. In the three generation case the neutrino oscillations are determined by two mass differences and three mixing angles neglecting the CP-violating phase. One of the mixing angles is irrelevant for solar neutrino problem [23,24] while being relevant to the atmospheric neutrino problem and one of the mass differences is constrained by the atmospheric neutrino deficit. Therefore the solar neutrino oscillations in the three flavour case

are dependent on three parameters. Because of the additional parameter, a larger region of the parameter space is allowed by the solar neutrino data compared to the two generation scenario. In section 2, we present the theoretical frame work for our analysis of the solar neutrino problem and in section 3 , we present the numerical results for the solar neutrino problem in conjunction with the atmospheric neutrino problem. The last section consists of a brief summary and discussion.

\section{THREE NEUTRINO OSCILLATIONS IN MATTER- A PERTURBATIVE ANALYSIS}

In this section we discuss the mixing between three flavours of neutrinos and obtain the probability for a $\nu_{e}$ produced in the sun to be detected as a $\nu_{e}$ on earth. The three flavour eigenstates are related to the three mass eigenstates in vacuum through a unitary 
transformation,

$$
\left[\begin{array}{c}
\nu_{e} \\
\nu_{\mu} \\
\nu_{\tau}
\end{array}\right]=U^{v}\left[\begin{array}{c}
\nu_{1}^{v} \\
\nu_{2}^{v} \\
\nu_{3}^{v}
\end{array}\right],
$$

where the superscript $v$ on r.h.s. stands for vacuum. The $3 \times 3$ unitary matrix $U^{v}$ can be parametrized by three Euler angles $(\omega, \phi, \psi)$ and a phase. The form of the unitary matrix can therefore be written in general as,

$$
U^{v}=U_{\text {phase }} \times U_{23}(\psi) \times U_{13}(\phi) \times U_{12}(\omega)
$$

where $U_{i j}\left(\theta_{i j}\right)$ is the mixing matrix between ith and jth mass eigenstates with the mixing angle $\theta_{i j}$. It has been shown that the expression for electron neutrino survival probability, integrated over the time of emission and of absorption, is independent of the phase and the third Euler angle $\psi 223,24$. They can be set to zero without loss of generality and we have the following form for $U^{v}$

$$
U^{v}=\left(\begin{array}{ccc}
c_{\phi} c_{\omega} & c_{\phi} s_{\omega} & s_{\phi} \\
-s_{\omega} & c_{\omega} & 0 \\
-s_{\phi} c_{\omega} & -s_{\phi} s_{\omega} & c_{\phi}
\end{array}\right)
$$

where $s_{\phi}=\sin \phi$ and $c_{\phi}=\cos \phi$ etc. The angles $\omega$ and $\phi$ can take values between 0 and $\pi / 2$. Note that one of the flavours decouples if either $\omega$ or $\phi$ is zero and we have a two flavour scenario. As mentioned earlier the approach here is similar to that of Joshipura and Krastev 26] who, however, assume that the mixing angle between second and third generation, $\psi$, is small and hence can be neglected. We wish to emphasise that this is not an assumption and infact $\psi$ can be arbitrary and the result for survival probability of the electron neutrino is independent of this 23,24. In fact the solution of the atmospheric neutrino deficit requires $\psi$ to be rather large. Together, solutions of the atmospheric neutrino deficit and the solar neutrino problem determine the mixing matrix $U^{v}$ completely apart from the CP-violating phase. 
The masses of the vacuum eigenstates are taken to be $\mu_{1}, \mu_{2}$ and $\mu_{3}$. In the mass eigenbasis, the (mass) $)^{2}$ matrix is diagonal,

$$
\begin{aligned}
M_{0}^{2}= & \left(\begin{array}{ccc}
\mu_{1}^{2} & 0 & 0 \\
0 & \mu_{2}^{2} & 0 \\
0 & 0 & \mu_{3}^{2}
\end{array}\right) \\
= & \mu_{1}^{2} I+\left(\begin{array}{lll}
0 & 0 & 0 \\
0 & \delta_{21} & 0 \\
0 & 0 & \delta_{31}
\end{array}\right),
\end{aligned}
$$

where $\delta_{21}=\mu_{2}^{2}-\mu_{1}^{2}$ and $\delta_{31}=\mu_{3}^{2}-\mu_{1}^{2}$. Without loss of generality, we can take $\delta_{21}$ and $\delta_{31}$ to be greater than zero. Neutrino oscillation amplitudes are independent of the first term so we drop it from further calculation. In the flavour basis the (mass) ${ }^{2}$ matrix has the form

$$
\begin{aligned}
M_{v}^{2} & =U^{v} M_{0}^{2} U^{v \dagger} \\
& =\delta_{31} M_{31}+\delta_{21} M_{21},
\end{aligned}
$$

where

$$
\begin{aligned}
M_{31} & =\left(\begin{array}{ccc}
s_{\phi}^{2} & 0 & s_{\phi} c_{\phi} \\
0 & 0 & 0 \\
s_{\phi} c_{\phi} & 0 & c_{\phi}^{2}
\end{array}\right) \\
M_{21} & =\left(\begin{array}{ccc}
c_{\phi}^{2} s_{\omega}^{2} & c_{\phi} s_{\omega} c_{\omega} & -c_{\phi} s_{\phi} s_{\omega}^{2} \\
c_{\phi} s_{\omega} c_{\omega} & c_{\omega}^{2} & -s_{\phi} s_{\omega} c_{\omega} \\
-c_{\phi} s_{\phi} s_{\omega}^{2} & -s_{\phi} s_{\omega} c_{\omega} & s_{\phi}^{2} s_{\omega}^{2}
\end{array}\right) .
\end{aligned}
$$

As in the two flavour case, matter effects can be included by adding $A(r)$, defined in (何), to the $e-e$ element of $M_{v}^{2}$. The matter corrected (mass) $)^{2}$ matrix in the flavour basis is

$$
M_{m}^{2}=\delta_{31} M_{31}+\delta_{21} M_{21}+A M_{A},
$$

where 


$$
M_{A}=\left(\begin{array}{lll}
1 & 0 & 0 \\
0 & 0 & 0 \\
0 & 0 & 0
\end{array}\right) .
$$

To calculate the evolution of a neutrino in matter we have to find the matter corrected eigenstates by diagonalizing $M_{m}^{2}$. For arbitrary values of $\delta_{31}$ and $\delta_{21}$, it is cumbersome to find the eigenvalues and eigenvectors of $M_{m}^{2}$ algebraically. However, the eigenvalue problem can be solved using perturbation theory, if the mass differences have the following hierarchy $\delta_{31} \gg \delta_{21}$. This assumption is plausible in light of the observed atmospheric muon neutrino deficit. Recently Kamiokande analyzed their atmospheric neutrino data, assuming that the deficit is caused by the oscillation of a $\nu_{\mu}$ into another flavour. Their analysis assumes mixing between only two flavours $\left(\nu_{\mu} \leftrightarrow \nu_{e}\right.$ or $\left.\nu_{\mu} \leftrightarrow \nu_{\tau}\right)$. For both cases their best fit yields a mass square difference of the order of $10^{-2} \mathrm{eV}^{2}$ [11]. In our analysis we take $\delta_{31}$ to be $10^{-2} \mathrm{eV}^{2}$. Thus we have $\delta_{31}$ much larger than $A_{\text {max }}$ and hence the oscillations involving the third generation are not influenced very much by the matter effects. In order for the matter effects to be significant (as necessitated by the solar neutrino problem), the other mass difference in the problem, $\delta_{21}$, should be such that the resonance condition is satisfied for some values of parameters. This means $\delta_{21} \sim A_{\max }$. Thus we work in an approximation where $\delta_{21}, A_{\max } \ll \delta_{31}$.

In this approximation, to the zeroth order, both the matter term and the term proportional to $\delta_{21}$ can be neglected in eq. (16). Then $M_{m}^{2}=\delta_{31} M_{31}$, whose eigenvalues and eigenvectors are

$$
\begin{gathered}
0 ;\left(\begin{array}{c}
c_{\phi} \\
0 \\
-s_{\phi}
\end{array}\right), \\
0 ;\left(\begin{array}{c}
0 \\
1 \\
0
\end{array}\right),
\end{gathered}
$$




$$
\delta_{31} ;\left(\begin{array}{c}
s_{\phi} \\
0 \\
c_{\phi}
\end{array}\right)
$$

Treating $A M_{A}+\delta_{21} M_{21}$ as perturbation to the dominant term in $M_{m}^{2}$ and carrying out degenerate perturbation theory, we get the matter dependent eigenvalues and eigenvectors,

$$
\begin{gathered}
m_{1}^{2} ;\left(\begin{array}{c}
c_{\phi_{m}} c_{\omega_{m}} \\
-s_{\omega_{m}} \\
-s_{\phi_{m}} c_{\omega_{m}}
\end{array}\right), \\
m_{2}^{2} ;\left(\begin{array}{c}
c_{\phi_{m}} s_{\omega_{m}} \\
c_{\omega_{m}} \\
-s_{\phi_{m}} s_{\omega_{m}}
\end{array}\right), \\
m_{3}^{2} ;\left(\begin{array}{c}
s_{\phi_{m}} \\
0 \\
c_{\phi_{m}}
\end{array}\right) .
\end{gathered}
$$

The above eigenvectors are the columns of the unitary matrix $U^{m}$ which relates the flavour eigenstates to matter dependent mass eigenstates $\nu_{i}^{m}$ through the relation

$$
\left[\begin{array}{c}
\nu_{e} \\
\nu_{\mu} \\
\nu_{\tau}
\end{array}\right]=U^{m}\left[\begin{array}{c}
\nu_{1}^{m} \\
\nu_{2}^{m} \\
\nu_{3}^{m}
\end{array}\right] .
$$

The matter dependent mixing angles can be expressed in terms of the vacuum parameters and $A$ as

$$
\begin{gathered}
\tan 2 \omega_{m}=\frac{\delta_{21} \sin 2 \omega}{\delta_{21} \cos 2 \omega-A \cos ^{2} \phi}, \\
\sin \phi_{m}=\sin \phi\left[1+\frac{A}{\delta_{31}} \cos ^{2} \phi\right] ; \cos \phi_{m}=\cos \phi\left[1-\frac{A}{\delta_{31}} \sin ^{2} \phi\right]
\end{gathered}
$$

The matter dependent eigenvalues $m_{i}^{2}$ are given by 


$$
\begin{aligned}
& m_{1}^{2}=A \cos ^{2} \phi \cos ^{2} \omega_{m}+\delta_{21} \sin ^{2}\left(\omega-\omega_{m}\right), \\
& m_{2}^{2}=A \cos ^{2} \phi \sin ^{2} \omega_{m}+\delta_{21} \cos ^{2}\left(\omega-\omega_{m}\right), \\
& m_{3}^{2}=\delta_{31}+A \sin ^{2} \phi \simeq \delta_{31} .
\end{aligned}
$$

$\omega_{m}$ can undergo a resonance if the values of $\delta_{21}, \phi$ and $\omega$ are such that the resonance condition

$$
A(r) \cos ^{2} \phi=\delta_{21} \cos 2 \omega
$$

is satisfied for some $r$. Note that this condition is very similar to the resonance condition in the two flavour case (eq. 6). The new feature here, which occurs due to the mixing among the three neutrino flavours, is the presence of the second mixing angle $\phi$ in the resonance condition. This dependence on $\phi$ leads to a larger region of allowed parameter space in the three flavour oscillation scenario as will be shown in the next section. Since $\delta_{21}, A(r)$ and $\cos ^{2} \phi$ are all positive, a resonance can occur only if $\cos 2 \omega$ is also positive, or if $\omega<\pi / 4$.

In the three flavour case, the electron neutrino survival probability is given by

$$
\left\langle P_{e e}\right\rangle=\sum_{i, j=1}^{3}\left|U_{e i}^{v}\right|^{2}\left|U_{e j}^{m}\right|^{2}\left|\left\langle\nu_{i}^{v} \mid \nu_{j}^{m}\right\rangle\right|^{2} \text {. }
$$

$\left|\left\langle\nu_{i}^{v} \mid \nu_{j}^{m}\right\rangle\right|^{2}$ is the probability that the $j$ th matter dependent eigenstate evolves into $i$ th vacuum eigenstate. As in the two flavour case, if the adiabatic approximation holds, then

$$
\left|\left\langle\nu_{i}^{v} \mid \nu_{j}^{m}\right\rangle\right|^{2}=\delta_{i j}
$$

We introduce the jump probabilities

$$
x_{i j}=\left|\left\langle\nu_{i}^{v} \mid \nu_{j}^{m}\right\rangle\right|^{2} \text { for } i \neq j
$$

to take into account the non-adiabatic transitions, if the adiabatic condition doesn't hold.

Because $\delta_{31} \gg A_{\max }, \delta_{21}$, the third eigenvalue, both in vacuum and in matter, is much larger than the other two eigenvalues. Non-adiabatic effects are significant only if the eigenvalues of two states come close together [28]. Therefore the jump probabilities involving the third state, $x_{13}$ and $x_{23}$ are expected to be negligibly small. Thus we have the expression for electron neutrino survival probability to be 


$$
\begin{aligned}
\left\langle P_{e e}\right\rangle= & \cos ^{2} \phi \cos ^{2} \phi_{m}\left(\cos ^{2} \omega \cos ^{2} \omega_{m}+\sin ^{2} \omega \sin ^{2} \omega_{m}\right)+\sin ^{2} \phi \sin ^{2} \phi_{m} \\
& -x_{12} \cos ^{2} \phi \cos ^{2} \phi_{m} \cos 2 \omega \cos 2 \omega_{m} .
\end{aligned}
$$

For $x_{12}$ we use the formula,

$$
x_{12}=\frac{\exp \left[-\frac{\pi \gamma F}{2}\right]-\exp \left[-\frac{\pi \gamma F}{2 \sin ^{2} \omega}\right]}{1-\exp \left[-\frac{\pi \gamma F}{2 \sin ^{2} \omega}\right]}
$$

where $\gamma$ is defined in equation (8) and

$$
F=1-\tan ^{2} \omega
$$

for an exponentially varying solar density [22]. We use this form for the jump probability since it is valid both for large and small mixing angles. In the extreme non-adiabatic limit $x_{12} \rightarrow \cos ^{2} \omega$ and when $\gamma F>>1$, we have the usual Landau-Zener jump probability given by $x_{12} \rightarrow \exp \left[-\frac{\pi \gamma F}{2}\right]$ as expected. Infact for much of the allowed parameter space, this form can be used without any appreciable change in the results obtained.

\section{RESULTS}

In this section we discuss the results of the numerical analysis first for the solar neutrino problem and using that we map out the region in the parameter space which contains the solution to the atmospheric neutrino problem.

\section{A. solar neutrinos}

We analyze the expression for $\left\langle P_{e e}\right\rangle$ in (28) and find the ranges of $\delta_{21}, \omega$ and $\phi$ allowed by the three solar neutrino experiments. Since $\delta_{31} \gg A_{\max }$, we see from the expression for $\phi_{m}$ in (22) that the angle $\phi$ is almost unaffected by the matter effects. However, $\omega_{m}$ can be significantly different from $\omega$ and can undergo resonance if the resonance condition in (24) is satisfied. Since this resonance condition depends on $\phi$, in addition to $\delta_{21}$ and $\omega$, a larger region of parameter space satisfies the three constraints from the experiments. 
To search for the regions allowed in the three parameter space $\delta_{21}, \omega$ and $\phi$, we define the suppression factors observed by the three types of experiments

$$
\begin{gathered}
y_{G a}=\frac{R_{G a ; a v g}}{R_{G a ; S S M}}=0.563 \pm 0.067, \\
y_{C l}=\frac{R_{C l}}{R_{C l ; S S M}}=0.318 \pm 0.051, \\
y_{K a m}=\frac{R_{\text {Kam }}}{R_{\text {Kam } ; S S M}}=0.51 \pm 0.07,
\end{gathered}
$$

where the first number refers to the average of the data given by two experiments- namely GALLEX and SAGE. The predicted SSM rates for various experiments were taken from Bahcall-Pinsonneault SSM calculations [3]. The uncertainties in $y_{i}$ are the sum of the experimental uncertainty in the numerator and the theoretical uncertainty in the denominator, added in quadrature.

The predictions for $y_{i}$ for the three flavour oscillation scenario are obtained by convoluting the SSM fluxes and the detector cross sections with $\left\langle P_{e e}\right\rangle$ from (28). The expression we use is

$$
y=\frac{\sum_{K} \int_{E_{\max }}^{E_{\max }} d E \Phi_{K}(E) \sigma(E)<P_{e e}>(E)}{\sum_{K} \int_{E_{\min }}^{E_{\max }} d E \Phi_{K}(E) \sigma(E)},
$$

where the sum over $\mathrm{K}$ refers to the neutrino fluxes from various sources contributing to the process. We also include the contributions from the $\mathrm{CNO}$ cycle apart from the dominant contributions from the p-p cycle. In the case of Kamioka, only the ${ }^{8} B$ flux contributes and one must also take into account the neutral current contribution arising from the muon neutrinos interacting with the detector material. The parameter ranges are then calculated by putting vetos on $y$ at $1 \sigma$ and $1.6 \sigma$ levels. The energy dependent fluxes were taken from Ref. [3] and the cross sections were taken from Ref. [29].

Figure 1 shows the allowed values of $\omega$ and $\phi$ with $\delta_{21}$ varying between $10^{-6} \mathrm{eV}^{2}$ and $10^{-4} \mathrm{eV}^{2}$. Note that the allowed values of $\delta_{21}$ are also determined by the same veto conditions. In the two generation case it is a standard practice to plot $\delta_{21}$ against $\sin ^{2}(2 \omega)$ since that is the combination that enters the survival probability. In the three generation 
case all possible circular functions of the mixing angles are possible. Hence we depart from the standard practice in this paper and plot the angles themselves. The points refer to the allowed values after the vetos corresponding to all three experiments are imposed. The dark squares show the values allowed by $1 \sigma$ uncertainties given in (31) whereas the hollow squares show the values allowed when the uncertainty is increased to 1.6 $\sigma$. Fig.2 shows the allowed regions in the $\phi-\delta_{21}$ plane, with $\omega$ varying between 0 and $\pi / 2$ but obeying the same set of vetos. In Figs. 1 and 2 if we restrict ourselves to the $\phi=0$ lines (the y-axes) we get the known [2] two-flavour solutions for $\omega$ and $\delta_{21}$. The large extended regions of the parameter space brought in through the additional degree of freedom $\phi$ in the three-flavour scenerio are shown clearly in Figs.1 and 2. For completeness we also plot in Fig. 3 the allowed range in the $\omega-\delta_{21}$ plane. Here again the three-flavour scenerio provides an enlargement of the allowed parameter space over that of the two-flavour solution (small regions around the isolated dark patch in the left and around the end of the dark arm on the right).

The various regions of the allowed parameter space may be classified as follows:

1. small $\delta_{21}$, small $\omega$, small $\phi$,

2. large $\delta_{21}$, large $\omega$, small $\phi$,

3. small $\delta_{21}$, small $\omega$, large $\phi$,

4. large $\delta_{21}$, small $\omega$, large $\phi$,

5. large $\delta_{21}$, large $\omega$, large $\phi$,

where the small or large $\delta_{21}$ means either $\delta_{21}<10^{-5} \mathrm{eV}^{2}$ or $\delta_{21}>10^{-5} \mathrm{eV}^{2}$. The first two regions corresponding to small $\phi$ in the above classification belong to an approximate two generation situation since the angle $\phi$ is small. The one corresponding to small $\omega$ is the usual non-adiabatic solution, whereas the one corresponding to large $\omega$ is the usual adiabatic solution. The rest invoke the genuine three generation oscillation mechanism. In the two flavour scenario, the small angle solution (corresponding to $\omega$ small as in case 1 above) 
gives the best fit [2]. There the parameter space allowed at $1 \sigma$ level is very small because the resonance condition and the non-adiabatic jump factor fix $\delta_{21}$ and $\omega$ almost uniquely. These values of parameters indicate that the neutrinos from the $p-p$ cycle suffer very little suppression and those from ${ }^{7} \mathrm{Be}$ suffer almost complete suppression as will be illustrated soon in the analysis of the survival probability.

In the three flavour scenario, the resonance condition (eq. 24) and the survival probability (eq. 28) are dependent on the second angle $\phi$ also. The suppression of the $p-p$ neutrinos depends on the value of $\phi$ and if this suppression is significant, then the complete suppression for ${ }^{7} \mathrm{Be}$ neutrinos can be relaxed. This is one of the important differences between the three flavour and the two flavour oscillations.

Figure 4 shows the energy dependence of $\left\langle P_{e e}\right\rangle$ for some representative values of $\omega, \phi$, and $\delta_{21}$. The curve labelled (a) corresponds to $\phi=2^{\circ}$. As there is very little mixing between the first and the third generation of neutrinos, this is infact an almost two generation case. In agreement with the two generation analysis, there is almost no supression of the $p-p$ neutrinos and the ${ }^{7} \mathrm{Be}$ neutrinos are almost completely suppressed. The survival probability at high energies relevant to Kamioka is almost a linear function with an average around 0.5 as one would expect. Also here the values of $\omega$ and $\delta_{21}$ are small (they are almost equal to the values obtained in the two flavour case) and the non-adiabatic effects become important beyond $2 \mathrm{MeV}$. Keeping $\omega$ small if we increase $\phi$ in the allowed region there is a perceptible reduction in the probability in the $p-p$ energy range and an increase in the survival probability of the ${ }^{7} B e$ neutrinos (curves (b) and (c)). When $\delta_{21}$ is increased, however, there is a qualitative change in the survival probability profile. In this range both $\omega$ and $\phi$ are allowed to be large. Here also there is a qualitative change when $\omega$ is small or large. For large $\omega$ the survival probability is a smooth function resembling the adiabatic case of the two generation analysis (curves (d) and (f)) whereas for small $\omega$ it is almost a step function (curve (e)) which is like the classic adiabatic case discussed by Bethe in the two generation case [19]. One common feature of the large $\delta_{21}$ case is that the $p-p$ neutrinos undergo substantial suppression varying between $0.6-0.5$. The resonance also occurs at a 
much higher energy than in the small $\delta_{21}$ case. Curve (f) has $\omega, \phi$ and $\delta_{21}$ all large and in some sense it can be called 'most representative' of the three flavour oscillation scenario because both the mixing angles in this case are large. In all the above cases, except (e), the average survival probability above $7 \mathrm{MeV}$ is in the neighbourhood of 0.4 which is what is required by the Kamioka data and there is no dramatic change from one to the other. This is not so at low energies where the curves differ dramatically. In this sense Kamioka experiment cannot distinguish between different theoretical scenarios of masses and mixings.

One way of experimentally measuring the energy dependence of $\left\langle P_{e e}\right\rangle$ is to look at the recoil electron spectrum in those detectors that use $\nu_{e}-e$ scattering. In Fig.5 we have shown the recoil electron spectrum for the six cases plotted in Fig. 4. Except case (f), they cannot be distinguished beyond $10 \mathrm{MeV}$, whereas there are substantial differences at low energies. While this energy range is not completely accessible in Kamioka, it is interesting to note that it may be possible to see this difference in the experimental recoil electron spectrum in the SNO [30] and Borexino [31] detectors. Note that in computing the recoil electron spectrum, we have used the spectrum of ${ }^{8} B$ neutrinos as input. This is because the threshold in experiments which can measure the recoil electron spectrum (like SNO and Kamioka) is more than a few MeVs where only this flux matters. The only exception is Borexino where the threshold is much lower and there are other contributions below $1.5 \mathrm{MeV}$. In particular the ${ }^{7} \mathrm{Be}$ neutrino source, which is a line spectrum at $0.862 \mathrm{MeV}$, will show up as a sharp bump in the recoil spectrum where the height of the bump depends on the survival probability. A complete absense of the bump would point to the set parameters as in case (a) of Fig.4.

Finally we consider a non-standard mixing which leads a substantial region in the parameter space. We consider a situation where the electron neutrino is coupled more strongly to the heavier mass eigenstate $\nu_{2}$. Obviously this implies that the mixing angle between the first two mass eigenstates $\omega$, is greater than $\pi / 4$. In the standard analysis the mixing has to be less than $\pi / 4$ so that the resonance condition is satisfied as can be seen from eq.(24). This is true in the two as well as in the three generation case since the LHS of the resonance 
condition is positive for arbitrary $\phi$ whereas the sign in RHS depends on the magnitude of $\omega$. There are no strong theoretical reasons not to consider this situation. Because $\omega_{m}$ at core is very close to $\pi / 2, \omega_{m}$ is then constrained to be $\omega \leq \omega_{m}<\pi / 2$. Infact for $\delta_{21} \leq 10^{-7} \mathrm{eV}^{2}, \omega_{m}$ is approximately $\pi / 2$. Since $\phi$ hardly varies with density the effective survival probability may be approximately written as,

$$
\left\langle P_{e e}\right\rangle=\cos ^{4} \phi \sin ^{2} \omega+\sin ^{4} \phi-x_{12} \cos ^{4} \phi|\cos 2 \omega|
$$

where we have retained the jump probability $x_{12}$. While it may appear some what unusual to keep the jump probability when there is no resonance, a plot of the eigenvalues clearly shows that the difference between the first two eigenvalues is not very different from that of the standard case close to vacuum and one cannot completely discard the existence of non-adiabatic jumps between mass eigenstates. However, most of the derivations of the jump probability assume the existence of resonance and the profile of the density variation close to resonance. Since we do not have a handle on this, we assume that the jump probability is simply given by $x_{12}=\exp (-C / E)$ and treat $\mathrm{C}$ as a free parameter of the theory. The survival probability is then energy dependent as would be required by the solution to the solar neutrino puzzle. The resulting parameter space is shown in Fig.6 for $\omega, \phi$. The parameter $\mathrm{C}$ varies from 0.4 to 6.3 in the allowed region. If we assume any one of the expressions for the jump probability discussed earlier, then we will have to discard small values of $\mathrm{C}(C<4)$ since then the jump probability becomes very large and unacceptable. However in the allowed region, the points corresponding to small $\mathrm{C}$ are very few and there is no substantial change from the plot shown in Fig.6. We also show some typical variation of the survival probability $\left\langle P_{e e}\right\rangle$ for some typical values of $\omega, \phi$ and $C$ in Fig.7. The curve (a) corresponds to small $\mathrm{C}$ where non-adiabatic effects are important while the curve (b) corresponds to large $\mathrm{C}$ which is an almost adiabatic case. We wish to stress that this is an adhoc solution but we have analysed this situation because there are no strong theoretical reasons to ignore this possibility.

To conclude this section, we note that the solution to the solar neutrino puzzle fixes 
the parameter space defined by $\omega, \phi$ and $\delta_{21}$. While we have actually chosen the fourth parameter $\delta_{31}$ to be $10^{-2} \mathrm{eV}^{2}$ we might as well have set the limit $\delta_{31}>10^{-3} \mathrm{eV}^{2}$ without affecting our results. One therefore requires more inputs to fix the range of $\delta_{31}$ and the angle $\psi$ (mixing angle between second and the third generation neutrinos) which is arbitrary as far as the solar neutrino puzzle is concerned. The new input is provided by the analysis of the atmospheric neutrino problem which we consider next.

\section{B. Atmospheric Neutrinos}

In order to fix the mixing matrix completely we still need to fix the range of $\psi$, which is the mixing angle between the second and third generation neutrinos, as this is arbitrary in the solar neutrino analysis. To have a consistent solution for both solar neutrino and the atmospheric neutrino problems, we need to show that there exists a range of $\psi$ in the allowed range of parameters occuring in the solar neutrino problem. To ensure this we first define the ratio

$$
R=\frac{\left(\frac{\phi_{\nu \mu}}{\phi \nu_{e}}\right)_{o b s}}{\left(\frac{\phi_{\nu \mu}}{\phi \nu_{e}}\right)_{M C}}
$$

which measures the ratio of the observed muon neutrino flux to the electron neutrino flux to that expected from Monte-Carlo calculations of neutrino production in the atmosphere. The most recent measurement of this ratio by the Kamiokande collaboration [11] yields

$R=0.57_{-0.07}^{+0.08} \pm 0.07$ in the multi-GeV range. The depletion is further confirmed by the observation of the zenith-angle dependence. The result for sub-GeV range atmospheric neutrinos is $R=0.60_{-0.05}^{+0.06}$ which is consistent with the multi-GeV range data. We may therefore assume that the suppression is approximately energy independent from sub-GeV to multi-GeV range of energies. Assuming that this depletion is due to the vacuum oscillations amongst the neutrino flavours, this ratio may be written as,

$$
R=\frac{P_{\mu \mu}+r P_{e \mu}}{P_{e e}+\frac{1}{r} P_{\mu e}},
$$


where $r=\phi_{\nu_{e}} / \phi_{\nu_{\mu}}=0.45$ is the ratio of the flux of electron neutrinos to that of muon neutrinos at the production point. Note that $r$ is simply the inverse of the flux ratio expected on the basis of the Monte-Carlo calculations (see eq.(34)). We now assume that the survival probabilities $\left(P_{e e}, P_{\mu \mu}\right)$ and the oscillation probability $\left(P_{\mu e}\right)$ are given by the full three generation mixing matrix defined by the angles $\phi, \omega, \psi$ and the two mass squared differences $\delta_{21}, \delta_{31}$. The vacuum oscillation probability between two flavours is then given by,

$$
\begin{aligned}
& P_{i j}=U_{i 1}^{2} U_{j 1}^{2}+U_{i 2}^{2} U_{j 2}^{2}+U_{i 3}^{2} U_{j 3}^{2}+2 U_{i 1} U_{i 2} U_{j 1} U_{j 2} \cos \left(2.53 \frac{d \delta_{21}}{E}\right) \\
& +2 U_{i 1} U_{i 3} U_{j 1} U_{j 3} \cos \left(2.53 \frac{d \delta_{31}}{E}\right)+2 U_{i 3} U_{i 2} U_{j 3} U_{j 2} \cos \left(2.53 \frac{d \delta_{32}}{E}\right)
\end{aligned}
$$

where $\mathrm{i}$ and $\mathrm{j}$ are the flavour indices, $\mathrm{E}$ is the energy given in units of $\mathrm{MeV}, \delta_{i j}$ is the mass differences in $e V^{2}$ and $d$ is the distance of traversal given in meters. These probabilities explicitly depend on the distance $d$ travelled by the neutrinos from the point of production to the point of detection and is approximately about 13,000 kms for the upward moving neutrinos. This distance is much less than the oscillation length between the first two generations( since $\delta_{21}$ is small). Therefore the cosine factor involving $\delta_{21}$ can be safely set equal to unity. As mentioned before, Kamioka has also observed that the level of suppression for the atmospheric muon neutrinos is approximately the same both for sub-GeV and the multi-GeV neutrinos. This can be ensured if the energy dependent factors involving $\delta_{31}$ and $\delta_{32}$ are such that the cosine functions can be replaced by the corresponding averages. This is possible if and only if many oscillation lengths are contained in the distance travelled by neutrinos to the detector. This then sets the limits on the mass squared difference $\delta_{31}>10^{-3} \mathrm{eV}^{2}$. The large $\delta_{31}$ regions $\left(\delta_{31}>10^{-1} \mathrm{eV}^{2}\right)$ are excluded at 90 percent C.L by the analysis of the multi-GeV neutrino data [11]. While we have used the central value $10^{-2} \mathrm{eV}^{2}$ in our solar neutrino analysis, the results for both solar and atmospheric neutrinos remain unchanged if the value is further increased and marginal changes occur for values close to $10^{-3} \mathrm{eV}^{2}$ because of the approximations we made in the solar neutrino analysis.

Therefore the only range to be fixed is for the mixing angle $\psi$. This we do by requiring the theoretical value of $\mathrm{R}$ calculated from eq.(35) is within $1 \sigma$ and $1.6 \sigma$ of the experimental 
value. The resulting range for $\psi$ is shown in Fig.8, whereas usual the full squares show the $1 \sigma$ veto and the open squares show $1.6 \sigma$ veto.

A few comments are in order here: As in the two generation analysis of the atmospheric neutrino problem, we find that the preferred values of $\psi$ is large and around $\pi / 4$. This can be checked easily by looking at the conversion probability $P_{\mu e}$ in the allowed range of parameters for the atmospheric neutrino problem. It turns out that this conversion probability is always less than twenty percent. Thus the solution to the atmospheric neutrino problem is mainly driven by the $\nu_{\mu}-\nu_{\tau}$ oscillations whereas the solution to the solar neutrino problem is mainly driven by $\nu_{e}-\nu_{\mu}$ oscillations at least for small values of $\phi$. However there are large domains of the parameter space where one requires the full three generation analysis presented here, to have a consistent solution to both the problems.

\section{SUMMARY AND DISCUSSION}

We have examined in detail the possible solutions to the solar neutrino and atmospheric neutrino puzzles in the realistic three generation framework. There are in general three mixing angles, one phase from the mixing matrix and two mass squared differences which define the full parameter space. In the case of solar neutrinos the survival probability for the electron neutrino, even after taking into account the matter effects, is independent of the phase and one of the mixing angles. We also fix one of the mass squared differences by appealing to the atmospheric neutrino problem. Thus our parameter space in the solar neutrino analysis consists of two angles and one mass squared difference. In our case these are chosen to be $\omega$ which gives the mixing between first and second generations , $\phi$ which is the mixing between first and third generations and $\delta_{21}$ which is the mass squared difference between the first two generations. The mass difference $\delta_{31}$ is fixed to be around $10^{-2} \mathrm{eV}^{2}$ to explain the atmospheric neutrino problem. We have mapped out the parameter $\operatorname{space}\left(\phi, \omega, \delta_{21}\right)$ by invoking the vetos arising from the data given by the three solar neu-

trino experiments. Next we have used these allowed ranges of parameters from the solar 
neutrino analysis as input in the atmospheric neutrino analysis to fix the angle $\psi$ and find that there exists a substantial range in this parameter which allows a solution to the atmospheric neutrino puzzle. The numerical calculations necessarily depend on the bin size for the parameters. We have ensured that the bin size we have chosen is such that a further reduction will not change the overall profile of the allowed region. However it is conceivable that the rough edges that one still sees in parts of the allowed region will be smoothed out by a further reduction of the bin size.

In conclusion, we have shown that there exists a consistent solution to the solar and atmospheric neutrino deficit puzzles within the framework of standard MSW mechanism based on the set of all available measurements of the solar neutrino fluxes. The full analysis involves five parameters which we have mapped out by accommodating the solar and atmospheric neutrino fluxes seen by the present set of experiments. While the allowed region in the parameter space is still large, these can be constrained further by measuring the distributions of recoil electron energies in solar neutrino detectors that use $\nu-e$ scattering. Although the threshold energy at the Kamioka detector is rather too high for this purpose, the SNO and Borexino detectors may be effective in narrowing the parameter space. Finally we would like to remark that the analysis of solar and atmospheric neutrino problems presented here is exploratory in nature. This is so since with time the errors are bound to change which inturn will affect the vetos imposed by us at $1 \sigma$ and $1.6 \sigma$ levels. Nevertheless we believe there is already sufficient indication that a robust solution of both problems is possible within the framework provided by the mechanism of neutrino oscillations with three generations.

Acknowledgements: This work was started during the Third Workshop on High Energy Particle Physics (WHEPP 3) in Madras. We gratefully acknowledge the collaboration in the initial stages with Prof.A.S.Joshipura. We also thank Prof. K.V.L.Sarma for keeping us informed of the recent developments in neutrino oscillations. 


\section{REFERENCES}

[1] K. V. L. Sarma, TIFR/TH/94-13 PUB, October 1994, (to appear in IJMP 10, 1995).

[2] P. Langacker, UPR-0640T, November 1994, hep-ph/9411339, Invited talk presented at 32nd International School of Subnuclear Physics Erice, July 1994 (hep-ph/9411339).

[3] J. N. Bahcall and M. H. Pinsonneault, Rev. Mod. Phys. 64, 885 (1992).

[4] K. Lande, Talk at Neutrino 94, at Eilat, Isreal, June 1994.

[5] M. Nakahata, Talk at International Conference on High Energy Physics, at Glasgow, August 1994.

[6] P. Anselman et al, Phys. Lett. B327, 377 (1994)

[7] V. Gavrin, Talk at International Conference on High Energy Physics, at Glasgow, August 1994.

[8] N. Hata and P. Langacker, Pennsylavania Preprint UPR-0625T, September 1994.

[9] W. Kwong and S. P. Rosen, Phys. Rev. Lett. 73, 369 (1994).

[10] Stephen Parke, Phys.Rev.Lett. 74, 839(1995).

[11] Kamiokande Collaboration: Y. Fukuda et al, Phys. Lett. B335, 237 (1994).

[12] D.Casper etal., Phys.Rev.Lett. 66, 2561(1991); R.Becker-Szendy et al., Phys. Rev. D 46, 3720(1992).

[13] M.Aglietta et al, Europhys. 8, 611(1989).

[14] Ch.Berger et al, Phys.Lett. B245, 305(1990).

[15] M.Goodman, Talk at the atmospheric neutrino workshop, Lousiana(1993).

[16] A.Acker, J.G.Learned, S.Pakvasa and T.J.Weiler, Phys.Lett.B298, 149(1993).

[17] S. M. Bilenky and B. Pontecorvo, Phys. Rep. 41, 225 (1978). 
[18] L. Wolfenstein, Phys. Rev. D17, 2369 (1978); D20, 2634 (1979)

S. P. Mikheyev and A. Yu. Smirnov, Yad. Fiz. 42, 1441 (1985) [Sov. J. Nucl. Phys. 42, 913 (1985)]; Nuovo Cimento, 9C, 17 (1986).

[19] H. A. Bethe, Phys. Rev. Lett. 56, 1305 (1986).

[20] J. N. Bahcall and H. A. Bethe, Phys. Rev. Lett. 65, 2233 (1990).

[21] N. Hata and P. Langacker, Phys. Rev. D50, 632 (1994).

[22] T. K. Kuo and J. Pantaleone, Rev. Mod. Phys. 61, 937 (1989).

[23] T. K. Kuo and J. Pantaleone, Phys. Rev. D35, 3432 (1987).

[24] A. S. Joshipura and M. V. N. Murthy, Phys. Rev. D37, 1374 (1988).

[25] David Harley, T.K.Kuo and J.Pantaleone, Phys.Rev.D47, 4059(1993).

[26] A.S.Joshipura and P.I.Krastev, Phys. Rev. D50, 3484 (1994).

[27] C.W.Kim and J.A.Lee, The Johns Hopkins University Preprint-JHU-TIPAC/930023, (hep-ph/9311317).

[28] L. D. Landau and E. M. Lifshitz, Non-relativistic Quantum Mechanics, 3rd Edition (1977), Pergamon Press.

[29] J. Bahcall, Neutrino Astrophysics, Cambridge University Press.

[30] G.T.Ewan et al., Sudbury Neutrino Observatory Proposal, Pub.No.SNO-87-12 (1987).

[31] "Borexino at Gran Sasso", Proposal for a real time detector for low energy solar neutrinos, Edited by G.Bellini, M.Campanella, D.Guigni and R.S.Raghavan (1991) 


\section{FIGURES}

FIG. 1. Allowed regions in $\phi-\omega$ plane (with $10^{-6} \mathrm{eV}^{2} \leq \delta_{21} \leq 10^{-4} \mathrm{eV}^{2}$ ) at $1 \sigma$ (dark squares) and at $1.6 \sigma$ (hollow squares).

FIG. 2. Allowed regions in $\phi-\log \left(\delta_{21} / \mathrm{eV}^{2}\right)$ plane (with $\left.0 \leq \omega \leq \pi / 2\right)$ at $1 \sigma$ (dark squares) and at $1.6 \sigma$ (hollow squares).

FIG. 3. Allowed regions in $\omega-\log \left(\delta_{21} / \mathrm{eV}^{2}\right)$ plane (with $\left.0 \leq \phi \leq \pi / 2\right)$ at $1 \sigma$ (dark squares) and at $1.6 \sigma$ (hollow squares).

FIG. 4. Survival probability $\left\langle P_{e e}\right\rangle$ vs $E_{\nu}$ for typical values of $\phi, \omega$ and $\delta_{21}$ in the allowed region. The parameters chosen are: (a) $\delta_{21}=4.0 \times 10^{-6}, \omega=2.5^{\circ}, \phi=2.0^{\circ}$; (b) $\delta_{21}=5.0 \times 10^{-6}, \omega=2.0^{\circ}, \phi=16.5^{\circ} ; \quad(\mathrm{c}) \delta_{21}=7.0 \times 10^{-6}, \omega=1.75^{o}, \phi=37.5^{\circ} ;$ (d) $\delta_{21}=2.5 \times 10^{-5}, \omega=35.0^{\circ}, \phi=3.0^{\circ} ; \quad(\mathrm{e}) \delta_{21}=7.0 \times 10^{-5}, \omega=2.0^{\circ}, \phi=30.0^{\circ} ;$ (f) $\delta_{21}=1.0 \times 10^{-4}, \omega=24.5^{\circ}, \phi=24.0^{\circ} ; \delta_{21}$ is given in terms of $\mathrm{eV}^{2}$.

FIG. 5. Recoil electron spectrum for different representative points of the allowed parameter region. The parameters for the differenct curves labelled (a)-(f) are the same as in Fig.4. The inset shows a comparison of all zix cases with the SSM spectrum(dashed line).

FIG. 6. Allowed regions in $\phi-\omega$ plane (with0.4 $\leq C \leq 6.4$ (dark squares) and at $1.6 \sigma$ (hollow squares) for the non-standard solutions.

FIG. 7. Typical survival probability profile in the non-standard case. The curve labelled (a) corresponds to $C=0.4, \omega=55^{\circ}, \phi=2^{\circ}$ and the curve labelled (b) corresponds to $C=6, \omega=89^{\circ}, \phi=38^{\circ}$.

FIG. 8. The allowed range of values for the mixing angle $\psi$ in the $\psi-\phi$ plane when the $\phi$ and $\omega$ are restricted to the range allowed by the solar neutrino problem(see Fig.1). 